\title{
FINITE-ELEMENT METHOD: ACCURACY AT A POINT
}

\author{
$\mathrm{BY}$ \\ ISAAC FRIED \\ Boston University
}

Introduction. The energy norm is the natural norm with which to work in the finiteelement (Rayleigh-Ritz) method, and it is already a classical result (see for instance [1]) that in this norm the error $\hat{e}=u-\hat{u}$ in the finite-element solution $\hat{u}$ is bounded by

$$
\|\hat{e}\|_{m} \leq c h^{p+1-m}\|u\|_{p+1}
$$

where $u, h, p, c$ and $m$ denote the exact solution, the diameter of the element, the degree of the complete polynomial in the shape functions, a positive coefficient independent of $h$ and $u$, and the order of the differential equation involved, respectively.

The passage from the readily available and general energy $\left(\|\cdot\|_{m}\right)$ error estimates to the uniform $\left(\|\cdot\|_{0}\right)$ error estimates can be accomplished via Rayleigh's principle. With this

$$
\|\hat{e}\|_{0} \leq \frac{1}{\omega}\|e\|_{m},
$$

$\omega$ being the fundamental frequency of the structure. But since $\|\cdot\|_{m}$ includes $m$ derivatives and $\|\cdot\|_{0}$ none, we suspect that Eq. (2) furnishes too pessimistic bounds. Indeed, by a clever trick, Nitsche [2] obtained, under some restrictions on the smoothness of the exact solution, a sharper bound on $\|\hat{e}\|_{0}$ than that obtained from Eq. (2).

Pointwise upper and lower bounds on the exact solution of second- and fourth-order problems in two and three dimensions were derived by Diaz and Greenberg [3, 4], Fujita [5], Maple [6], Washizu [7], Prager [8, p. 350], and Bramble and Payne [20]. These bounds were derived with the essential help of Green's function, and are expressed in terms of approximate solutions obtained with the Rayleigh-Ritz method using the principles of minimum potential energy and minimum complementary energy (or any admissible functions for these principles).

Concerning the pointwise error bounds on the finite-element (Rayleigh-Ritz) solution, Kantorovich and Krylov [9] obtained for the two-dimensional Dirichlet (fixedmembrane) problem solved with polynomial trial functions

$$
\|\hat{e}\|_{\infty} \leq O\left(\|\hat{e}\|_{1} \log ^{1 / 2}\|\hat{e}\|_{1}\right)
$$

where for the domain $D$

$$
\|\hat{e}\|_{\infty}=\sup _{D}|\hat{e}| .
$$

Shultz [10] inferred from this that for a rectangular domain discretized with multivariate

* Received March 12, 1972; revised version received December 20, 1972. 
elements of order $p$ (shape functions include a complete polynomial of degree $p$ )

$$
\|\hat{e}\|_{\infty} \leq O\left(h^{p} \log ^{1 / 2} h\right)
$$

which for $p=1$ agrees (except for $\log ^{1 / 2} h$ ) with the result of Nitsehe [11].

Making use of Sebolev's embedding theorems, Mikhlin [12] and Zlamal [13] obtained for the plate-bending problems $(m=2)$ that

$$
\|\hat{e}\|_{\infty} \leq c\|\hat{e}\|_{2}
$$

and therefore according to Eq. (1)

$$
\|\hat{e}\|_{\infty} \leq O\left(h^{p-1}\right) .
$$

Johnson and McLay [14] proved that for the two-dimensional elasticity problem in a rectangular domain discretized by bilinear $(p=1)$ elements, the error in the nodal displacements is bounded by $O\left(h^{1 / 2}\right)$. In this paper we:

i. derive pointwise bounds on the error in the finite-element solution and its derivatives (displacements and strains) in the interior of linear, bilinear and quadratic elements employed in the discretization of Poisson's equation in two and three dimensions.

ii. extend Markov's theorem [15] to two and three dimensions. This will permit us to relate the pointwise error in the displacements to the pointwise error in the strains.

iii. derive pointwise bounds on $\hat{e}$ and its derivatives in the case of second-order problems in two and three dimensions.

iv. discuss the pointwise accuracy of the finite element solution in the presence of singularities.

v. derive a general bound on $|u(P)-\hat{u}(P)|$ in terms of $\|u-\hat{u}\|_{m}$ and $\|v-\hat{v}\|_{m}$, $v$ being the response function to a unit impulse (point load) at $P$.

vi. apply this bound to obtain better pointwise bounds on $\hat{e}$, in the case of a thin plate, than that given by Eq. (7).

vii. show that with bilinear $(p=1)$ elements the best $\|\cdot\|_{1}$ approximation to a quadratic polynomial and with bicubic $(p=3)$ elements the best $\|\cdot\|_{2}$ approximation to a quartic polynomial are the interpolates (i.e. no error at the nodes). This indicates that with these elements a higher rate of convergence can be expected for the nodal values than for $\|\hat{e}\|_{\infty}$.

2. Energy theorems. The problems best suited for the method of finite elements are those which can be variationally expressed as the search for the minimum of the quadratic functional (say total potential energy)

$$
\pi(v)=\frac{1}{2} a(v, v)-(f, v)
$$

over the class of functions $v$ satisfying some essential (kinematic) boundary conditions, and for which $a(v, v)<\infty$. In the case of a fixed membrane, for instance, occupying the domain $D$

$$
(u, v)=\int_{D}\left(u_{x} v_{x}+u_{y} v_{v}\right) d x d y,
$$

whereas for the simply supported, clamped or free plate

$$
a(u, v)=\int_{D}\left(u_{x x} v_{x x}+2 u_{x y} v_{x y}+u_{y y} v_{y y}\right) d x d y .
$$


In both cases

$$
(u, v)=\int_{D} u v d x d y
$$

Since $a(v, v)$ is positive definite for all functions $v$ excluding the rigid-body modes, we can choose it as a norm

$$
a(v, v)=\|v\|_{m}^{2} .
$$

The minimization of $\pi(v)$ yields

$$
a(u, v)=(f, v)
$$

where, again, $u$ denotes the exact solution and $v$ any function satisfying the essential boundary conditions for which $a(v, v)<\infty$. Eq. (13) is the mathematical statement of the principle of virtual displacements. If $\hat{u}$ denotes the finite element solution, then corresponding to Eq. (13) we also have

$$
a(\hat{u}, \tilde{v})=(f, \tilde{v}) .
$$

Eqqs. (13) and (14) result in

$$
a(u-\hat{u}, \tilde{v})=0
$$

and in

$$
\begin{aligned}
& \pi(\hat{u})=-\frac{1}{2} a(\hat{u}, \hat{u})=-\frac{1}{2}(f, \hat{u}), \\
& \pi(u)=-\frac{1}{2} a(u, u)=-\frac{1}{2}(f, u),
\end{aligned}
$$

yielding

$$
\pi(\hat{u})-\pi(u)=\frac{1}{2} a(u-\hat{u}, u-\hat{u})
$$

from which we get

$$
(f, u) \geq(f, \hat{u})
$$

Choosing $f$ in Eq. (18) to be a point load (a delta function, an impulse) at $P$, we get from this equation that

$$
u(P) \geq \hat{u}(P) .
$$

The Cauchy-Schwarz inequality

$$
|a(u, v)| \leq\|u\|_{m}\|v\|_{m}
$$

is also of central importance here. Using this inequality, we prove the following theorem.

Theorem. Consider the $2 m$ th-order elliptic equation

$$
L u=f \quad \text { in } D,
$$

$u$ satisfying:

i. some essential (kinematic) boundary conditions on $\partial D_{1}$.

ii. some natural (dynamic) boundary conditions on $\partial D_{2}$. Consider also the auxiliary value problem 


$$
L v=\delta(P) \text { in } D
$$

where $\delta(P)$ is a unit impulse (point load) at $P$ and where $v$ satisfies the condition that integration by parts of $a(u-\hat{u}, v)$ gives rise to no boundary terms. If $\hat{u}$ and $\hat{v}$ are the finite-element solutions of $u$ and $v$, then

$$
|u(P)-\hat{u}(P)| \leq\|u-\hat{u}\|_{m}\|v-\hat{v}\|_{m} .
$$

Proof. According to Eq. (15),

$$
a(u-\hat{u}, v)=a(u-\hat{u}, v-\hat{v}) .
$$

Hence from Eq. (20) we get

$$
|a(u-\hat{u}, v)| \leq\|u-\hat{u}\|_{m}\|v-\hat{v}\|_{m} .
$$

Integrating $a(u-\hat{u}, v)$ by parts while recalling the particular choice of homogenous boundary conditions imposed on $v$ leads to

$$
|(u-\hat{u}, L v)| \leq\|u-\hat{u}\|_{m}\|v-\hat{v}\|_{m},
$$

from which Eq. (23) follows.

3. Second-order problems, pointwise convergence of displacements and strains in the interior of the elements. Consider the boundary-value problem

$$
\begin{aligned}
u_{x x}+u_{y y}=0 \quad \text { in } \quad D, \\
u=u^{*} \text { on } \quad \partial D,
\end{aligned}
$$

Suppose that this has been approximately solved with finite elements and that the solutions thus obtained is $\hat{u}$. We wish to bound $\hat{e}=u-\hat{u}$ as well as $\hat{e}_{x}$ and $\hat{e}_{y}$. To this end we write Eq. (20) in the form

$$
|a(\hat{e}, v)| \leq\|\hat{e}\|_{1}\|v\|_{1} .
$$

We draw in $D$ an interior circle of radius $a$ occupying the domain $\omega$ (see Fig. 1) and choose $v$ in Eq. (28) to be such that

$$
\begin{array}{ll}
v_{x x}+v_{y y}=0 & \text { in } \quad D-\omega, \\
v_{x x}+v_{y y}=1 / \pi a^{2} & \text { in } \omega .
\end{array}
$$

Since $\hat{e}=0$ on $\partial D$, integration by parts of $a(\hat{e}, v)$ results in

$$
\left|\frac{1}{\pi a^{2}} \int_{\omega} \hat{e} d x d y\right| \leq\|\hat{e}\|_{1}\|v\|_{1} .
$$

Let $\omega$ be confined now to the interior of linear or bilinear elements. In this case both $u$ and $\hat{u}$ are harmonic inside $\omega$ and by the mean value theorem we get that at the center $C$ of $\omega$

$$
|\hat{e}(C)| \leq\|\hat{e}\|_{1}[1+4 \log (d / a)]^{1 / 2} /(8 \pi)^{1 / 2}
$$

where $d$ denotes the diameter of $D$. For the center $C$ of an inscribed circle we thus get

$$
|\hat{e}(C)| \leq O\left(h \log ^{1 / 2} h\right) .
$$




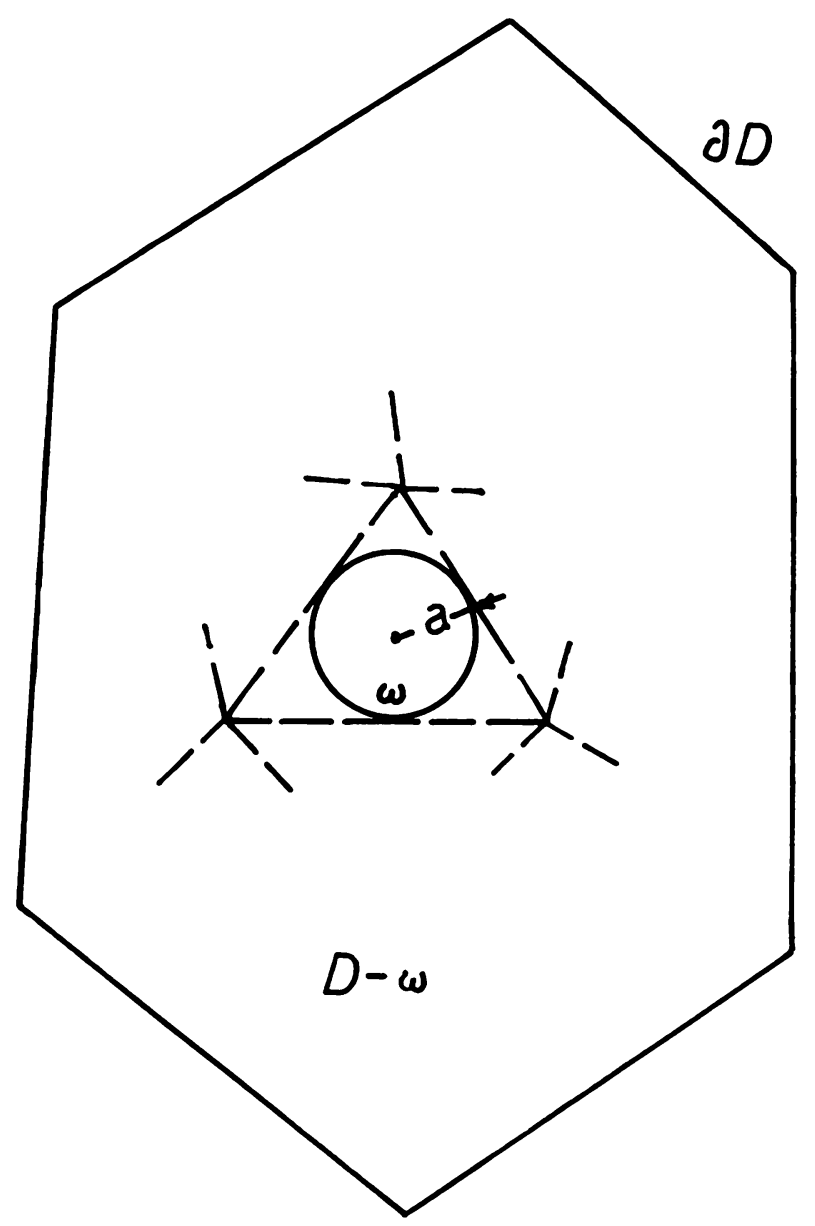

FIG. 1. The domain $D$ is triangulated. A circle of radius $a$ occupying the domain $\omega$ is inscribed inside an element.

The treatment of the corresponding three-dimensional problem

$$
\begin{aligned}
& u_{x x}+u_{y y}+u_{z z}=0 \text { in } D, \\
& u=u^{*} \text { on } \partial D,
\end{aligned}
$$

is entirely analogous to that of the plane problem except that in this case the circle is replaced by a sphere of radius $a$ and $v$ is chosen so as to satisfy

$$
\begin{array}{ll}
v_{x x}+v_{y y}+v_{z z}=0 & \text { in } D-\omega, \\
v_{x x}+v_{y y}+v_{z z}=-3 / 4 \pi a^{3} & \text { in } \omega .
\end{array}
$$

It follows that if the sphere $\omega$ is confined to the interior of linear or trilinear elements, then at the center $C$

$$
|\hat{e}(C)| \leq\|\hat{e}\|_{1}(3 d / 10 a-1 / 4)^{1 / 2} /(8 d)^{1 / 2},
$$

or at the center of an inscribed circle, 


$$
|\hat{e}(C)| \leq O\left(h^{1 / 2}\right) .
$$

For bounding $\hat{e}_{x}$ (or $\hat{e}_{y}$ ) we start with

$$
\left|\left(\hat{e}_{x}, v\right)\right| \leq\left\|\hat{e}_{x}\right\|_{0}\|v\|_{0}
$$

which, since $\left\|e_{x}\right\|_{0} \leq\|\hat{e}\|_{1}$, becomes

$$
\left|\left(\hat{e}_{x}, v\right)\right| \leq\|\hat{e}\|_{1}\|v\|_{0} .
$$

We choose $v$ to be

$$
\begin{array}{ll}
v=1 / \omega & \text { inside } \omega, \\
v=0 & \text { outside } \omega,
\end{array}
$$

$\omega$ being the volume of a sphere of radius $a$ inside $D$. In the case of quadratic $(p=2)$ elements both $u_{x}$ and $\hat{u}_{x}$ are harmonic inside the element and we get from Eqs. (38) and (39) that for the center $C$ of an inscribed circle in the plane

$$
|D \hat{e}(C)| \leq O(h),
$$

whereas for the inscribed sphere in three-dimensional second-order elements

$$
|D \hat{e}(C)| \leq O\left(h^{1 / 2}\right),
$$

in which $D$ stands for first-order differentiation.

4. Pointwise convergence of the finite-element solution and its derivatives in twoand three-dimensional second-order problems. Before proceeding to the pointwise estimates we need to extend Markov's theorem to two and three dimensions. This theorem states that if $u$ is a polynomial of degree $n$ in the interval $\left(x_{0}, x_{1}\right)$ then the derivative $u_{x}$ of $u$ is bounded by

$$
\left\|u_{x}\right\|_{\infty} \leq \frac{2 n^{2}}{h}\|u\|_{\infty}
$$

where $h=x_{1}-x_{0}$ and

$$
\|u\|_{\infty}=\sup _{x_{0} \leq x \leq x_{1}}|u| .
$$

Let $u(x, y)$ be a polynomial of degree $n$ in the trapezoid as in Fig. 2a with a small basis $h^{\prime}$. We will prove here that

$$
\left\|u_{x}\right\|_{\infty} \leq \frac{2 n^{2}}{h^{\prime}}\|u\|_{\infty} .
$$

For this we introduce the notation $\|u\|_{y, \infty}$ meaning $\|u\|_{\infty}$ on $y=$ constant. If $\left\|u_{x}\right\|_{\infty}$ occurs at the point $(x, y)$ then

$$
\left\|u_{x}\right\|_{\infty} \leq \frac{2 n^{2}}{h}\|u\|_{y, \infty}
$$

But since $\|u\|_{\infty} \geq\|u\|_{\nu, \infty}$ and since $h^{\prime} \leq h$ we readily get from Eq. (45) the result in Eq. (44).

From the trapezoid we pass on to the triangle and divide it into three trapezoids $A, B, C$ as in Fig. 2b, with bases $h_{1}$ and $h_{1} / 2, h_{2}$ and $h_{2} / 2, h_{3}$ and $h_{3} / 2$. The remaining 

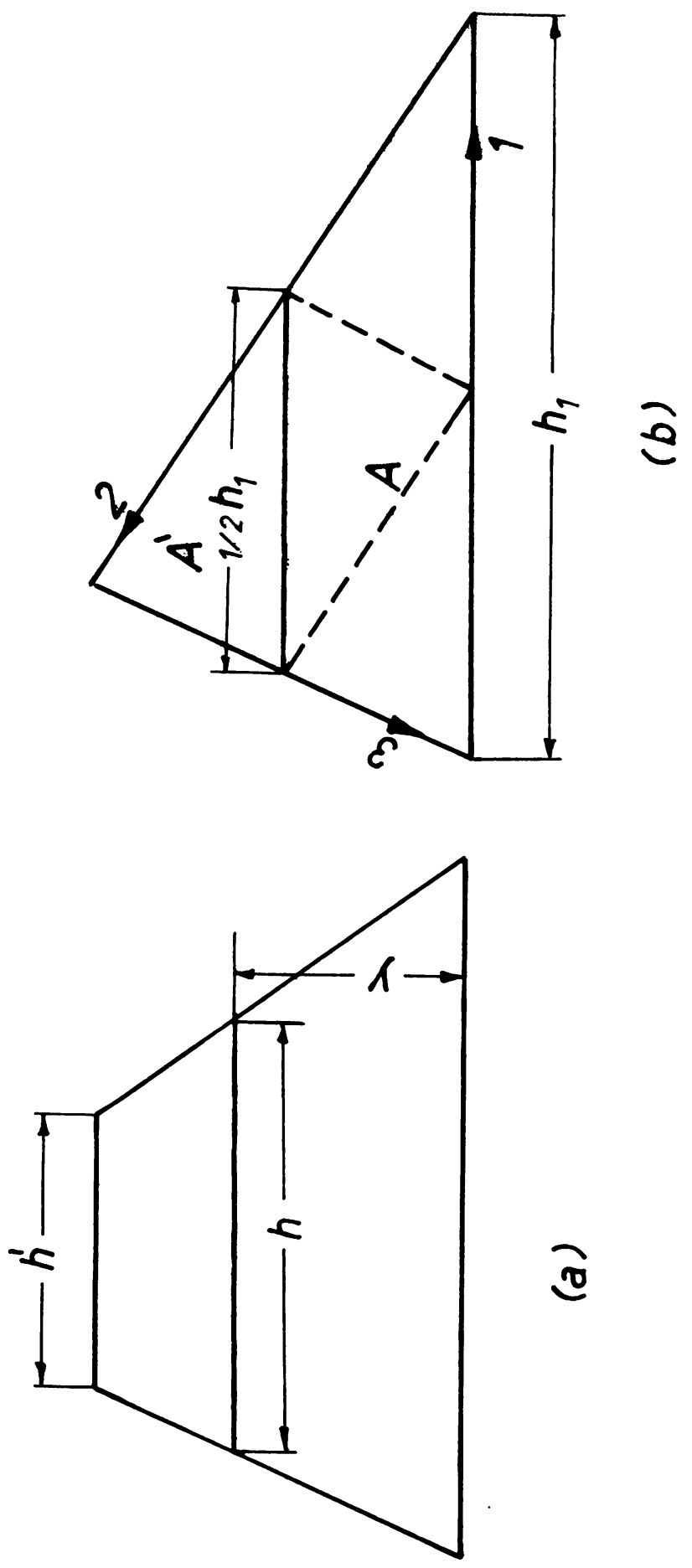

Fig. 2. (a) Trapezoidal domain with small basis $h^{\prime}$ and (b) a triangular domain divided into a trapezoid $A$ and a triangle $A^{\prime}$. 
three triangles with bases $h_{1} / 2, h_{2} / 2$ and $h_{3} / 2$ we denote by $A^{\prime}, B^{\prime}$ and $C^{\prime}$. We also introduce the three directions 1,2 and 3 parallel to the sides of the original triangles and seek to bound the three derivatives $u_{, i}, i=1,2,3$, in these three directions. Since all three directions are equivalent we concentrate on only one of them, say 3 . From Eq. (44) we have that

$$
\begin{aligned}
&\left\|u_{, 1}\right\|_{A, \infty} \leq \frac{4 n^{2}}{h_{1}}\|u\|_{A, \infty}, \\
&\left\|u_{, 2}\right\|_{B, \infty} \leq \frac{4 n^{2}}{h_{2}}\|u\|_{B, \infty}, \\
&\left\|u_{, 3}\right\|_{C, \infty} \leq \frac{4 n^{2}}{h_{3}}\|u\|_{C, \infty},
\end{aligned}
$$

where $\|u\|_{A, \infty}$ means $\|u\|_{\infty}$ in $A$. Also

$$
\|u\|_{C, \infty} \leq\|u\|_{\infty},\|u\|_{B, \infty} \leq\|u\|_{\infty} \text { and }\|u\|_{A, \infty} \leq\|u\|_{\infty}
$$

and since $C^{\prime} \subset A, C^{\prime} \subset B$

$$
\left\|u_{, 2}\right\|_{C^{\prime}, \infty} \leq\left\|u_{, 2}\right\|_{B, \infty},\left\|u_{, 1}\right\|_{C^{\prime}, \infty} \leq\left\|u_{, 1}\right\|_{A, \infty} .
$$

If we denote by $\alpha_{i}$ the interior angle between directions $i$ and $j$, then

$$
u_{, 3}=\left(\sin \alpha_{23} u_{, 1}+\sin \alpha_{13} u_{, 2}\right) / \sin \alpha_{12},
$$

and therefore

$$
\left\|u_{, 3}\right\|_{c^{\prime}, \infty} \leq\left(\left\|u_{, 1}\right\|_{c^{\prime}, \infty}+\left\|u_{, 2}\right\|_{c^{\prime}, \infty}\right) /\left|\sin \alpha_{12}\right|,
$$

and consequently we get from Eq. (48) that

$$
\left\|u_{, 3}\right\|_{c^{\prime}, \infty} \leq \frac{4 n^{2}}{\left|\sin \alpha_{12}\right|}\left(\frac{1}{h_{1}}+\frac{1}{h_{2}}\right)\|u\|_{\infty} .
$$

Since $\left\|u_{, 3}\right\|_{\infty}$ can be either in $C$ or $C^{\prime}$, Eqs. (46) and (51) result in

$$
\left\|u_{. i}\right\|_{\infty} \leq \frac{8 n^{2}}{\sin \theta} \frac{1}{h}\|u\|_{\infty} \quad i=1,2,3
$$

where $h$ and $\theta$ are the smallest side and angle, respectively, in the triangle. We draw attention once more to the fact that in Eq. (52) $u$ is a polynomial of degree $n$.

Further extension of Markov's theorem to the third dimension is entirely analogous to that into two dimensions and we do not intend to dwell on it here.

We use Eq. (52) to bound the derivatives of $\hat{e}$ in terms of $\hat{e}$ and $h$. By Taylor's theorem the exact solution $u$ can be separated into

$$
u=P+R \text { and } u_{x}=P_{x}+R^{\prime}
$$

where $P$ is a polynomial of degree $q$ and $R$ and $R^{\prime}$ the remainders. Since the finiteelement solution $\hat{u}$ is polynomial inside each element we have that

$$
\left\|\hat{u}_{x}-P_{x}\right\|_{\infty} \leq \frac{c}{h}\|\hat{u}-P\|_{\infty}
$$

where $c$ is a positive coefficient independent of $h$. Eq. (54), combined with Eqs. (52) 
and (53), leads to

$$
\left\|u_{x}-\hat{u}_{x}\right\|_{\infty} \leq \frac{c}{h}\|u-\hat{u}\|_{\infty}+\frac{c}{h}\|R\|_{\infty}+\left\|R^{\prime}\right\|_{\infty},
$$

or, with the notation $D^{m} u$ for the $m$ th derivative of $u$,

$$
\|R\|_{\infty} \leq c h^{a+1}\left\|D^{a+1} u\right\|_{\infty},\left\|R^{\prime}\right\|_{\infty} \leq c h^{a}\left\|D^{a+1} u\right\|_{\infty} .
$$

Eq. (55) can be rewritten now concisely as

$$
\|D \hat{e}\|_{\infty} \leq c_{1} h^{-1}\|\hat{e}\|_{\infty}+c_{2} h^{a}\left\|D^{a+1} u\right\|_{\infty} .
$$

This equation is of central importance here since it permits us to bound the error in the derivatives (strains) in term of the error in the displacement $u$.

Having established the bound in Eq. (57) we proceed to the estimation of $\|\hat{e}\|_{\infty}$. Consider the second-order boundary value problem

$$
\begin{gathered}
-\sum_{i, j=1}^{3} \frac{\partial}{\partial x_{i}} k_{i j} \frac{\partial u}{\partial x_{i}}+b u=f \text { in } D, \\
u=u^{*} \text { on } \partial D
\end{gathered}
$$

with $b \geq 0$ and $k_{i i}$ positive definite. Here

$$
a(u, v)=\int_{D}\left(\sum_{i, j=1}^{3} k_{i j} u_{x i} v_{x i}+b u v\right) d V .
$$

By the argument of the previous section we obtain also here

$$
\left|\frac{1}{\omega} \int_{\omega} \hat{e} d V\right| \leq\|\hat{e}\|_{1}\|v\|_{1}
$$

where $\omega$ is again a sphere of radius $a$ centered around $C$. Since $\hat{e}$ is continuous we can expand it around $C$ in the form

$$
\hat{e}=\hat{e}(C)+r \hat{e}_{r}(\rho)
$$

where $0 \leq \rho \leq r$. Thus

$$
\left|\frac{1}{\omega} \int_{\omega} \hat{e} d V\right| \leq\left|\hat{e}(C)+\frac{1}{\omega} \int_{\omega} r \hat{e}_{r} d V\right|,
$$

or

$$
\left|\frac{1}{\omega} \int_{\omega} \hat{e} d V\right| \geq|\hat{e}(C)|-\left|\frac{1}{\omega} \int_{\omega} r \hat{e}_{r} d V\right|,
$$

and consequently

$$
|\hat{e}(C)| \leq\|\hat{e}\|_{1}\|v\|_{1}+a\|D \hat{e}\|_{\omega, \infty} .
$$

If the sphere is allowed to wander over the complete domain $D$ (there is no problem at the boundary since $\hat{e}$ can be extended outside by $\hat{e}=0$ ) then with Eq. (57) we get

$$
\|\hat{e}\|_{\infty} \leq\|\hat{e}\|_{1}\|v\|_{1}+c_{1} a h^{-1}\|\hat{e}\|_{\infty}+c_{2} a h^{a}\left\|D^{a+1} u\right\|_{\infty} \text {. }
$$

Suppose first that $u$ is regular enough that $\left\|D^{p} u\right\|_{\infty}<\infty$. We choose then $q+1=p$ 
and $c_{1} a h^{-1}=\frac{1}{2}$, and since $\|\hat{e}\|_{1}\|v\|_{1}=O\left(h^{p} \log ^{1 / 2} h\right)$, Eq. (65) yields

$$
\|\hat{e}\|_{\infty} \leq O\left(h^{p} \log ^{1 / 2} h\right)
$$

in the plane, and

$$
\|\hat{e}\|_{\infty} \leq O\left(h^{p-1 / 2}\right)
$$

in space.

In order to observe what happens in the case of a singularity consider the case $u=O\left(r^{1 / 2}\right)$. The full energy rate of convergence can be regained in this case by quadratically varying the mesh size around the singularity [16]. If we denote by $N_{\text {r the }}$ radial number of elements then

$$
\|\hat{e}\|_{1}\|v\|_{1} \leq O\left(N_{r}^{-p} \log ^{1 / 2} a\right) .
$$

The smallest mesh size is $O\left(N_{r}^{-3}\right)$ and therefore

$$
\|\hat{e}\|_{\infty} \leq c_{1} N_{r}^{-p} \log a+c_{2} a N_{r}^{-3}\|\hat{e}\|_{\infty}+c_{3} a N_{r}^{-3 a}\left\|D^{a+1} u\right\|_{\infty} .
$$

Since we assumed $u=O\left(r^{1 / 2}\right)$ we select $q+1=0, a=N_{r}^{-(p+3)}$ and obtain from Eq. (69) that

$$
\|\hat{e}\|_{\infty} \leq O\left(N_{r}^{-p} \log ^{1 / 2} N_{r}\right),
$$

which means that $\hat{e}$ converges everywhere even at the singular point. Concerning the convergence of $D \hat{e}$ (i.e. strains and stresses), we see from Eq. (57) that even though $\|D \hat{e}\|_{\infty}$ becomes unbounded at the singularity the bound on it decreases as we move away from the singular point (i.e. if $\left\|D^{a+1} u\right\|_{\infty}$ is replaced by $\left\|D^{a+1} u\right\|_{D^{\prime}, \infty}, D^{\prime}$ being the domain $D$ minus the neighborhood of the singular point).

5. Fourth-order problems. For the clamped plate problem

$$
\begin{aligned}
\nabla^{4} u=f & \text { in } \quad D, \\
u=0, u_{n}=0 & \text { on } \quad \partial D,
\end{aligned}
$$

where $n$ is normal to $\partial D$, we have

$$
v=r^{2} \log r+g
$$

where $g$ is regular. It can be shown by direct computations (see also [16]) that for this $v$

$$
\|v-\hat{v}\|_{2} \leq \operatorname{ch} \log ^{1 / 2} h
$$

and we thus obtain from Eq. (23) that if the shape functions inside the element include a complete polynomial of degree $p$ then

$$
\|\hat{e}\|_{\infty} \leq O\left(h^{p} \log ^{1 / 2} h\right) .
$$

If $u$ is regular we also obtain from Eq. (57) that

$$
\left\|D^{m} \hat{e}\right\|_{\infty} \leq O\left(h^{p-m} \log ^{1 / 2} h\right) .
$$

6. Accuracy of nodal values. The discretization-energy error analysis is essentially reduced [17] to the question of how well an element of order $p$ (shape functions include 
a complete polynomial of degree $p$ ) can approximate a polynomial of degree $p+1$. It is appropriate therefore to introduce the best energy fit-the closest an element of order $p$ can energetically approach a polynomial of degree $p+1$ (or any other smooth function) without any interelement continuity constraints.

To fix ideas consider first a first-order string element inside which the displacement function $\tilde{u}$ is given by

$$
\tilde{u}=a_{0}+a_{1} x, \quad 0 \leq x \leq h .
$$

Since $\tilde{u}$ is linear we assume the true solution $u$ to be the quadratic $u=\alpha x^{2} / 2$ and seek the best energy fit by minimizing

$$
\|u-\tilde{u}\|_{1}{ }^{2}=\int_{0}^{h}\left(a_{1}-\alpha x\right)^{2} d x
$$

with respect to $a_{1}$. This minimization yields $a_{1}=\alpha h / 2$ and the best energy fit is with the element parallel to the interpolate, and

$$
\min \|u-\tilde{u}\|_{1}^{2}=\frac{1}{12} \alpha^{2} h^{3} .
$$

It is not difficult to show that the best energy fit in the case of a spring is with the elements parallel to the interpolate. Indeed, by minimizing

$$
\|u-\tilde{u}\|_{1}^{2}=\int_{x_{1}}^{x_{2}}\left(u_{x}-a_{1}\right)^{2} d x
$$

with respect to $a_{1}$ we get

$$
a_{1}=\left(u\left(x_{2}\right)-u\left(x_{1}\right)\right) /\left(x_{2}-x_{1}\right) .
$$

For the cubic beam element with

$$
\tilde{u}=a_{0}+a_{1} x+a_{2} x^{2}+a_{3} x^{3}, \quad 0 \leq x \leq h,
$$

the best energy fit is obtained by minimizing

$$
\|u-\tilde{u}\|_{2}^{2}=\int_{0}^{h}\left(u_{x x}-2 a_{2}-6 a_{3} x\right)^{2} d x
$$

with respect to both $a_{2}$ and $a_{3}$, yielding also that here the best energy fit is the interpolate such that at the nodal points $u=\tilde{u}$ and $u_{x}=\tilde{u}_{x}$. Choosing $u$ in Eq. (82) to be the quartic $u=\alpha x^{4} / 24$ we get

$$
\min \|u-\tilde{u}\|_{2}^{2}=\frac{1}{720} \alpha^{2} h^{4} .
$$

Since the interpolate is variationally admissible and since the finite-element solution is, according to Eq. (17), the best in the energy, the actual finite-element solutions for the beam and string will be the interpolates with no discretization errors $[18,19]$ for the nodal values $u$ and $u_{x}$.

The displacement $\tilde{u}$ inside a bilinear membrane element is given by

$$
\tilde{u}=a_{0}+a_{1} x+a_{2} y+a_{3} x y, \quad 0 \leq x \leq h, \quad 0 \leq y \leq h,
$$

and we assume therefore that

$$
u=\frac{1}{2} \alpha_{0} x^{2}+\alpha_{1} x y+\frac{1}{2} \alpha_{2} y^{2} .
$$


The best energy fit in this case is obtained from the minimization of

$$
\|u-\tilde{u}\|_{1}^{2}=\int_{0}^{h} \int_{0}^{h}\left[\left(\alpha_{0} x+\alpha_{1} y-a_{1}-a_{3} y\right)^{2}+\left(\alpha_{1} x+\alpha_{2} y-a_{2}-a_{3} x\right)^{2}\right] d x d y
$$

with respect to $a_{1}, a_{2}$ and $a_{3}$, yielding

$$
a_{1}=\frac{1}{2} \alpha_{0} h, a_{2}=\frac{1}{2} \alpha_{2} h, a_{3}=\alpha_{1} .
$$

Thus also for the bilinear element the best energy fit to the quadratic is the interpolate with no errors at the nodes, and

$$
\min \|u-\tilde{u}\|_{2}^{2}=\frac{1}{12} h^{4}\left(\alpha_{0}^{2}+\alpha_{2}^{2}\right) .
$$

Repeating the same analysis for the first-order triangular, first-order membrane element, we readily verify that for this element the best energy fit is not the interpolate.

The bicubic plate bending element (with four nodal points at the corners and with the four nodal values $u, u_{x}, u_{y}, u_{x y}$ at each node) includes a complete polynomial of the third degree. We therefore assume $u$ to be the quartic

$$
u=\frac{1}{24} \alpha_{0} x^{4}+\frac{1}{2} \alpha_{1} x^{3} y+\frac{1}{2} \alpha_{2} x^{2} y^{2}+\frac{1}{2} \alpha_{3} x y^{3}+\frac{1}{24} \alpha_{4} y^{4} .
$$

The same computations that we carried out earlier in this section yield also that for this element the best energy fit to the quartic is the interpolate such that $u=\tilde{u}, u_{x}=\tilde{u}_{x}$, $u_{y}=\tilde{u}_{y}$ and $u_{x y}=\tilde{u}_{x y}$ at the corners, and

$$
\min \|u-\tilde{u}\|_{2}^{2}=\frac{1}{72} \delta h^{6}\left(\alpha_{0}{ }^{2}+\alpha_{4}{ }^{2}\right) .
$$

We conclude from the analysis of this section that:

i. In bilinear membrane elements the rate of convergence of the nodal values can be expected to be higher than that of $\|\hat{e}\|_{\infty}$.

ii. The nodal accuracy of the bilinear element is higher than that of the first-order triangular element.

iii. The rate of convergence of $u, u_{x}, u_{y}$ and $u_{x y}$ at the nodes of bicubic plate bending element can be expected to be higher than that of $\|\hat{e}\|_{\infty}$.

iv. The points where the most accurate stresses occur can be found by locating the points where the derivatives in the best energy fit agree with those in the fitted-to higher-order polynomial. In tensor product elements these happen to be Gauss points.

\section{REFERENCES}

[1] G. Strang, Approximation in the finite element method, Numer. Math. 19, 81-98 (1972)

[2] J. Nitsche, Ein Kriterium fur die Quasi-Optimalitat des Ritschen Verfahrens, Numer. Math. 11, 346-348 (1968)

[3] J. B. Diaz and H. J. Greenberg, Upper and lower bounds for the solution of the first boundary value problem of elasticity, Quart. Appl. Math. 6, 326-331 (1948)

[4] J. B. Diaz and H. J. Greenberg, Upper and lower bounds for the solution of the first biharmonic boundary value problem, J. Math. Phys. 27, 193-201 (1948)

[5] H. Fujita, Contribution to the theory of upper and lower bounds in boundary value problems, J. Phys. Soc. Japan 10, 1-8 (1955)

[6] C. G. Maple, The Dirichlet problem: bounds at a point for the solution and its derivatives, Quart. Appl. Math. 8, 213-228 (1950)

[7] K. Washizu, Bounds for solutions of boundary value problems in elasticity, J. Math. Phys. 32, 117-128 (1953)

[8] J. L. Synge, The hypercircle in mathematical physics, Cambridge University Press, 1957 
[9] L. V. Kantorovich and V. I. Krylov, Approximate methods of higher analysis, Interscience Publishers, 1958

[10] M. H. Shultz, Multivariate spline functions and elliptic problems, in Approximations with special emphasis on spline functions, Academic Press, 1969

[11] J. Nitsche, Linearer Spline-Functionen und die Methoden von Ritz fur elliptische Randwertprobleme, Arch. Rat. Mech. Anal. 36, 348-355 (1970)

[12] S. G. Mikhlin, Variational methods in mathematical physics, Pergamon Press, Oxford, 1964

[13] M. Zlamal, On the finite element method, Numer. Math. 12, 394-409 (1968)

[14] M. W. Johnson, Jr. and R. W. McLay, Convergence of the finite element method in the theory of elasticity, J. Appl. Mech. 35, 274-278 (1968)

[15] E. Hill, G. Szegö and J. D. Tomarkin, On some generalizations of a theorem by A. Markoff, Duke Math. J. 3, 729-739 (1937)

[16] I. Fried and Shok Keng Yang, Best finite elements distribution around a singularity, AIAA J. 10, 1244-1246 (1972)

[17] G. Strang, The finite element method and approximation theory, in Numerical solution of partial differential equations II, ed. B. Hubbard, 1971

[18] F. V. Filho, Comment on 'Computation of stress resultants from element stiffness matrices', AIAA J. 6, 571-572 (1968)

[19] B. L. Hulme, Interpolation by Ritz approximation, J. Math. Mech. 18, 337-341 (1968)

[20] J. H. Bramble and L. E. Payne, Pointwise bounds in the first biharmonic boundary value problem, J. Math. Phys. 42, 278-286 (1963) 